\title{
WZW superconformal blocks from three dimensions
}

\author{
I.N. McArthur \\ II. Institut für Theoretische Physik, Universität Hamburg, Luruper Chaussee 149, W-2000 Hamburg 50, FRG
}

Received 16 April 1991

\begin{abstract}
A three-dimensional theory whose canonical quantization yields wavefunctions which can be identified with the superconformal blocks of two-dimensional super Wess-Zumino-Witten theory is presented. In particular, the anomalous Ward identities satisfied by the superconformal blocks in the presence of a supergauge background are reproduced in terms of constraints obeyed by the wavefunctions of the three-dimensional theory. The action contains the bosonic Chern-Simons action but does not possess a three-dimensional supersymmetry. The "time" coordinate plays the role of an interpolating parameter between two-dimensional superconnections. The structure of the Hilbert space of the theory is examined.
\end{abstract}

\section{Introduction}

New insights into the nature of two-dimensional conformal field theories have arisen recently as a result of a three-dimensional interpretation of these theories. As shown by Witten [1] and elaborated on by many groups [2], the conformal blocks of a two-dimensional conformal field theory (2D CFT) can be realized as wavefunctions for a canonically quantized three-dimensional Chern-Simons theory (3D CS). In particular, the Verlinde operators [3] have a very natural interpretation in this picture.

It is natural to inquire whether a similar correspondence exists between the superconformal blocks for superconformal field theories and wavefunctions for some three-dimensional theory. The first idea that springs to mind is to supersymmetrize the 3D CS theory. However, one immediately encounters the problem that the lagrangian for a supersymmetric theory is not invariant under supersymmetry but transforms by a total derivative, which means that the action is not supersymmetric on a bounded manifold. This results in the failure of the wavefunction for 3D supersymmetric CS theory to be invariant under supersymmetry transformations, which makes an identification with the manifestly supersymmetric superconformal blocks problematic (for attempts in this direction, see ref. [4], and additional remarks in ref. [5]).

However, it is not immediately obvious that 3D supersymmetry is the relevant structure to consider. The relation between even-dimensional gauge theories and Chern-Simons terms in one dimension higher is well established in topological approaches to nonabelian anomalies, in which the extra dimension plays the role of an interpolating parameter [6]. In a certain sense, it is the nonabelian anomaly which is the key to the link between the conformal blocks for the Wess-Zumino-Witten models and 3D CS theory. The anomalous Ward identity obeyed by the conformal blocks implies that they can be considered as sections of a holomorphic line bundle over the space of chiral connections modulo (complexified) gauge transformations. The wavefunctions for the canonical quantization of 3D CS theory are, following the general principles of Kähler quantization, also sections of a holomorphic line bundle. The relation between the theories arises through the equivalence of the line bundles.

In supersymmetric gauge theories, there is also a relation between even-dimensional nonabelian anomalies and CS-like terms involving a bosonic interpolating parameter [7]. However, the supersymmetry does not extend to the "extra dimension" afforded by the interpolating parameter. Thus, if a 3D theory is to reproduce the anomalous Ward identities of super Wess-Zumino-Witten theory, it could be expected that it need not involve 
3D supersymmetry. In this paper, we present a proposal for such a theory. Indeed, the theory even fails to be Lorentz invariant in $3 \mathrm{D}$, and so is best thought of as a quantum mechanical model with a supersymmetry defined on the configuration space.

\section{Superconformal blocks for SWZW theory}

We begin with a review of $N=1$ super Wess-Zumino-Witten (SWZW) theory on a Riemann surface $\Sigma$, and construct the corresponding superconformal blocks. A supergravity background on this surface determines a superconformal structure specified by local superconformal coordinates $(z, \theta, \bar{z}, \bar{\theta})=(Z, \bar{Z})[8]$. For a superfield $\mathscr{G}(Z, \bar{Z})$ taking values in a compact simple Lie group G, the level $k$ SWZW action takes the form [9]

$$
\begin{aligned}
& S_{\mathrm{WZW}}[\mathscr{G}]=\frac{\mathrm{i} k}{4 \pi} \int \mathrm{d} z \wedge \mathrm{d} \bar{z} \int \mathrm{d}^{2} \theta\left(\mathscr{G}^{-1} \mathrm{D}_{\theta} \mathscr{G}, \mathscr{G}^{-1} \mathrm{D}_{\bar{\theta}} \mathscr{G}\right) \\
& \quad+\frac{i k}{4 \pi} \int \mathrm{d} t \int \mathrm{d} z \wedge \mathrm{d} \bar{z} \int \mathrm{d}^{2} \theta\left(\tilde{\mathscr{G}}^{-1} \partial_{t} \tilde{\mathscr{G}},\left\{\tilde{\mathscr{G}}^{-1} \mathrm{D}_{\theta} \overline{\mathscr{G}}, \overline{\mathscr{G}}^{-1} \mathrm{D}_{\bar{\theta}} \tilde{\mathscr{G}}\right\}\right),
\end{aligned}
$$

where $\mathrm{D}_{\theta}=\partial_{\theta}+\theta \partial_{z}, \int \mathrm{d}^{2} \theta \bar{\theta} \theta=1,($,$) denotes an inner product on the Lie algebra \mathrm{g}$ of $\mathrm{G}$ invariant under the adjoint action, and $\widetilde{G}(t, Z, \bar{Z})$ is the extension of $\mathscr{G}(Z, \bar{Z})$ to a $3 \mathrm{D}$ disc with boundary $\Sigma$ and local coordinates $(t, z, \bar{z})$. The second term in the action is topological, in that under an infinitesimal variation $\delta \tilde{G}$ of the extension,

$$
\begin{aligned}
& \delta\left(\tilde{\mathscr{G}}^{-1} \partial_{t} \tilde{\mathscr{G}},\left\{\tilde{\mathscr{G}}^{-1} \mathrm{D}_{\theta} \tilde{\mathscr{G}}, \widetilde{\mathscr{G}}^{-1} \mathrm{D}_{\bar{\theta}} \tilde{\mathscr{G}}\right\}\right)=\partial_{\ell}\left(\widetilde{\mathscr{G}}^{-1} \delta \tilde{\mathscr{G}},\left\{\tilde{\mathscr{G}}^{-1} \mathrm{D}_{\theta} \widetilde{\mathscr{G}}_{\mathscr{G}^{-1}} \mathrm{D}_{\bar{\theta}} \tilde{\mathscr{G}}\right\}\right) \\
& -\mathrm{D}_{\tilde{\theta}}\left(\tilde{\mathscr{G}}^{-1} \delta \tilde{\mathscr{G}},\left[\tilde{\mathscr{G}}^{-1} \partial_{t} \tilde{\mathscr{G}}, \tilde{\mathscr{G}}^{-1} \mathrm{D}_{\theta} \tilde{\mathscr{G}}\right]\right)-\mathrm{D}_{\theta}\left(\tilde{\mathscr{G}}^{-1} \delta \tilde{\mathscr{G}},\left[\tilde{\mathscr{G}}^{-1} \partial_{t} \tilde{\mathscr{G}}, \tilde{G}^{-1} \mathrm{D}_{\bar{\theta}} \tilde{\mathscr{G}}\right]\right) \text {, }
\end{aligned}
$$

a total derivative. In fact, with the parametrization

$$
\tilde{\mathscr{G}}=\tilde{g} \exp [\mathrm{i}(\theta \tilde{\psi}+\tilde{\theta} \tilde{\bar{\psi}}+\bar{\theta} \theta \tilde{F})],
$$

all dependence on $\tilde{\psi}, \tilde{\psi}$, and $\tilde{F}$ in the topological term in the action appears in the form of total derivatives and so is independent of their extension; the remaining manifestly $3 \mathrm{D}$ piece is

$$
-\frac{i k}{4 \pi} \int \mathrm{d} t \int \mathrm{d} z \wedge \mathrm{d} \bar{z}\left(\tilde{g}^{-1} \partial_{t} \tilde{g},\left[\tilde{g}^{-1} \partial_{z} \tilde{g}, \tilde{g}^{-1} \partial_{z} \tilde{g}\right]\right) \text {. }
$$

This is the usual topological term for the bosonic WZW theory, and the action is in general not the same for extensions $\tilde{g}$ which are not homotopic. However, if $2 k /(\psi, \psi)$ is an integer (with $\psi$ the highest root of $\mathrm{g}$ ), then the functional integral is well defined $[10]$ due to the proportionality of the difference of the topological term for two extensions to a $3 \mathrm{D}$ winding number.

The action (1) satisfies a Polyakov-Wiegmann condition [11],

$$
S_{\mathrm{wZW}}[\mathscr{H} \mathscr{G}]=S_{\mathrm{WZW}}[\mathscr{H}]+S_{\mathrm{WZW}}[\mathscr{G}]+\frac{\mathrm{i} k}{2 \pi} \int \mathrm{d} z \wedge \mathrm{d} \bar{z} \int \mathrm{d}^{2} \theta\left(\mathrm{D}_{\theta} \mathscr{G} \cdot \mathscr{G}-1, \mathscr{H}^{-1} \mathrm{D}_{\bar{\theta}} \mathscr{H}\right) .
$$

A gauge field background is specified by $\mathrm{g}^{\mathbb{C}}$-valued superfields $\mathscr{A}_{\theta}$ and $\mathscr{A}_{\hat{\theta}}$ with $\mathscr{A}_{\theta}=-\left(\mathscr{A}_{\hat{A}}\right)^{*}$. These superfields can be written locally in the form $\mathscr{A}_{\bar{\theta}}=\mathscr{K}^{-1} \mathrm{D}_{\bar{\theta}} \mathscr{K}$ and $\mathscr{A}_{\theta}=\mathscr{K}^{*} \mathrm{D}_{\theta} \mathscr{K}^{*-1}$ for a superfield $\mathscr{K}$ taking values in $\mathrm{G}^{\mathrm{C}}$ [12]; only if $\mathscr{K}^{*}=\mathscr{K}^{-1} \in \mathrm{G}$ is the connection flat. The gauged SWZW action is [13]

$$
\begin{aligned}
& S_{\mathrm{WZW}}\left[\mathscr{G}, \mathscr{A}_{\theta}, \mathscr{A}_{\bar{\theta}}\right]=S_{\mathrm{WZW}}\left[\mathscr{K} \mathscr{G} \mathscr{K}^{*}\right]-S_{\mathrm{WZW}}\left[\mathscr{K}^{*}\right] \\
& \quad=S_{\mathrm{WZW}}[\mathscr{G}]+\frac{\mathrm{i} k}{2 \pi} \int \mathrm{d} z \wedge \mathrm{d} \bar{z} \int \mathrm{d}^{2} \theta\left[\left(\mathrm{D}_{\theta} \mathscr{G} \cdot \mathscr{G}^{-1}, \mathscr{A}_{\bar{\theta}}\right)-\left(\mathscr{A}_{\theta}, \mathscr{G}^{-1} \mathrm{D}_{\bar{\theta}} \mathscr{G}\right)-\left(\mathscr{A}_{\theta}, \mathscr{G}^{-1} \mathscr{A}_{\bar{\theta}} \mathscr{G}\right)+\left(\mathscr{A}_{\theta}, \mathscr{A}_{\bar{\theta}}\right)\right] .
\end{aligned}
$$


Under the $G^{\mathbb{C}}$-valued gauge transformation $[14,15]$

$$
\mathscr{G} \rightarrow \mathscr{H}^{-1} \mathscr{G}_{\mathscr{H}^{*-1}}, \quad \mathscr{A}_{\theta} \rightarrow \mathscr{A}_{\theta}^{* *-1}=\mathscr{H}^{*}\left(\mathscr{A}_{\theta}+\mathrm{D}_{\theta}\right) \mathscr{H}^{*-1}, \quad \mathscr{A}_{\bar{\theta}} \rightarrow \mathscr{A}_{\hat{\theta}}^{*}=\mathscr{H}^{-1}\left(\mathscr{A}_{\theta}+\mathrm{D}_{\bar{\theta}}\right) \mathscr{H}
$$

it is easily verified using the first expression in (4) that

$$
\begin{aligned}
& S_{\mathrm{WZW}}\left[\mathscr{H}^{-1} \mathscr{G}^{*-1}, \mathscr{A}_{\theta}^{* *-1}, \mathscr{A}_{\theta}^{\mathscr{H}}\right]=S_{\mathrm{WZW}}\left[\mathscr{G}, \mathscr{A}_{\theta}, \mathscr{A}_{\theta}\right]-S_{\mathrm{WZW}}[\mathscr{H}]-S_{\mathrm{WZW}}\left[\mathscr{H}^{*}\right] \\
& +\frac{\mathrm{i} k}{2 \pi} \int \mathrm{d} z \wedge \mathrm{d} \bar{z} \int \mathrm{d}^{2} \theta\left[-\left(\mathrm{D}_{\theta} \mathscr{H} \cdot \mathscr{H}^{-1}, \mathscr{A}_{\bar{\theta}}\right)-\left(\mathscr{A}_{\theta}, \mathrm{D}_{\bar{\theta}} \mathscr{H}^{*-1} \cdot \mathscr{H}^{*}\right)+\left(\mathscr{A}_{\theta}^{* *-1}, \mathscr{A}_{\bar{\theta}}^{*}\right)-\left(\mathscr{A}_{\theta}, \mathscr{A}_{\bar{\theta}}\right)\right] .
\end{aligned}
$$

The action is invariant under the diagonal subgroup of gauge transformations with $\mathscr{H}=\mathscr{H}^{*-1} \in \mathrm{G}$.

The effective action $\Gamma\left[\mathscr{A}_{\theta}, \mathscr{A}_{\bar{\theta}}\right]$ is defined by

$$
\exp \left(-\Gamma\left[\mathscr{A}_{\theta}, \mathscr{A}_{\theta}\right]\right)=\int[\mathrm{d} \mathscr{G}] \exp \left(-S_{\mathrm{WZW}}\left[\mathscr{G}, \mathscr{A}_{\theta}, \mathscr{A}_{\theta}\right]\right)
$$

where $\int[\mathrm{d} \mathscr{G}]$ denotes the functional integral over the component fields of $\mathscr{G}$. We assume that $\int[\mathrm{d} \mathscr{G}]$ is regulated in a manner which preserves supersymmetry and so that $\int\left[\mathrm{d}\left(\mathscr{H}^{-1} \mathscr{G}_{\mathscr{H}^{*-1}}\right)\right]=\int[\mathrm{d} \mathscr{G}]$. This is justified because the gauge transformations act vectorially on the chiral fermionic components of $\mathscr{G}$ so there is no anomaly (it is also consistent with the usual OPE for the super Kac-Moody currents $\mathscr{H}_{\theta}(Z)=-k \mathrm{D}_{\theta} \mathscr{G} \cdot \mathscr{G}^{-1}$ which follows from the Ward identity below with $\mathscr{A}_{\theta}=0$ ). It then follows from (5) and (6) that

$$
\begin{aligned}
& \Gamma\left[\mathscr{A}_{\theta}^{* *-1}, \mathscr{A}_{\bar{\theta}}^{\mathscr{H}}\right]=\Gamma\left[\mathscr{A}_{\theta}, \mathscr{A}_{\bar{\theta}}\right]-S_{\mathrm{WZW}}[\mathscr{H}]-S_{\mathrm{WZW}}\left[\mathscr{H}^{*}\right] \\
& \quad+\frac{\mathrm{i} k}{2 \pi} \int \mathrm{d} z \wedge \mathrm{d} \bar{z} \int \mathrm{d}^{2} \theta\left[-\left(\mathrm{D}_{\theta} \mathscr{H}^{*} \cdot \mathscr{H}^{-1}, \mathscr{H}_{\bar{\theta}}\right)-\left(\mathscr{A}_{\theta}, \mathrm{D}_{\bar{\theta}} \mathscr{H}^{*-1} \cdot \mathscr{H}^{*}\right)+\left(\mathscr{A}_{\theta}^{* *-1}, \mathscr{A}_{\theta}^{\mathscr{H}}\right)-\left(\mathscr{A}_{\theta}, \mathscr{A}_{\bar{\theta}}\right)\right],
\end{aligned}
$$

so that one can identify $\exp \left(-\Gamma\left[\mathscr{A}_{\theta}, \mathscr{A}_{\theta}\right]\right)$ with a sum of terms of the form

$$
\exp \left(-\Gamma_{+}\left[\mathscr{A}_{\theta}\right]\right) \exp \left(-\Gamma_{-}\left[\mathscr{A}_{\theta}\right]\right) \exp \left(-\frac{\mathrm{i} k}{2 \pi} \int \mathrm{d} z \wedge \mathrm{d} \bar{z} \int \mathrm{d}^{2} \theta\left(\mathscr{A}_{\theta}, \mathscr{A}_{\hat{\theta}}\right)\right)
$$

(corresponding to a modular invariant combination of superconformal blocks), where $\Gamma_{+}$and $\Gamma_{-}$obey the Ward identities

$$
\begin{aligned}
& \Gamma_{+}\left[\mathscr{A}_{\theta}^{\mathscr{*}}\right]=\Gamma_{+}\left[\mathscr{A}_{\theta}\right]-S_{\mathrm{WZW}}[\mathscr{H}]-\frac{\mathrm{i} k}{2 \pi} \int \mathrm{d} z \wedge \mathrm{d} \bar{z} \int \mathrm{d}^{2} \theta\left(\mathrm{D}_{\theta} \mathscr{H} \cdot \mathscr{H}^{-1}, \mathscr{A}_{\bar{\theta}}\right), \\
& \Gamma_{-}\left[\mathscr{A}_{\theta}^{* *}\right]=\Gamma_{-}\left[\mathscr{A}_{\theta}\right]-S_{\mathrm{WZW}}\left[\mathscr{H}^{-1}\right]-\frac{\mathrm{i} k}{2 \pi} \int \mathrm{d} z \wedge \mathrm{d} \bar{z} \int \mathrm{d}^{2} \theta\left(\mathscr{A}_{\theta}, \mathrm{D}_{\bar{\theta}} \mathscr{H} \cdot \mathscr{H}^{-1}\right) .
\end{aligned}
$$

The chiral partition functions $Z_{+}\left[\mathscr{A}_{\theta}\right]=\exp \left(-\Gamma_{+}\left[\mathscr{A}_{\theta}\right]\right)$ and $Z_{-}\left[\mathscr{A}_{\bar{\theta}}\right]=\exp \left(-\Gamma_{-}\left[\mathscr{A}_{\hat{\theta}}\right]\right)$ in the presence of background gauge superfields will be referred to as the holomorphic and antiholomorphic superconformal blocks respectively (so named because the gauge superfields couple to the holomorphic and antiholomorphic super Kac-Moody currents respectively).

For an infinitesimal gauge transformation $\mathscr{H}=\exp (\mathrm{i} 4),(8)$ takes the form

$$
\delta_{A} \Gamma_{+}\left[\mathscr{A}_{\theta}\right]=\frac{k}{2 \pi} \int \mathrm{d} z \wedge \mathrm{d} \bar{z} \int \mathrm{d}^{2} \theta\left(\mathrm{D}_{\theta} \Lambda, \mathscr{A}_{\theta}\right) .
$$

Since $\delta_{A} \mathscr{A}_{\bar{\theta}}=\mathrm{i} \mathscr{Y}_{\bar{\theta}} A \equiv \mathrm{iD} \bar{D}_{\bar{\theta}} A+\mathrm{i}\left[\mathscr{A}_{\bar{\theta}}, A\right]$, this is equivalent to

$$
\mathscr{P}_{\theta} \frac{\delta \Gamma_{+}\left[\mathscr{A}_{\theta}\right]}{\delta \mathscr{A}_{\theta}^{q}}=-\frac{k}{\pi}\left(\mathrm{D}_{\theta} \mathscr{A}_{\theta a}\right)
$$

where the supercurrent $\delta \Gamma\left[\mathscr{A}_{\hat{\theta}}\right] / \delta \mathscr{A}_{\theta}^{a}$ is defined using 
$\frac{\delta}{\delta \mathscr{A}_{\bar{\theta}}^{a}(Z, \bar{Z})} \mathscr{A}_{\theta}^{b}(W, \bar{W})=\delta_{a}^{b} \delta^{(2)}(z-w) \delta^{(2)}\left(\theta-\theta^{\prime}\right)$,

with $W=\left(w, \theta^{\prime}\right)$ and

$\frac{1}{2} \mathrm{i} \int \mathrm{d} z \wedge \mathrm{d} \bar{z} \int \mathrm{d}^{2} \theta \delta^{(2)}(z-w) \delta^{(2)}\left(\theta-\theta^{\prime}\right)=1$.

Rewritten in terms of the holomorphic superconformal block, the infinitesimal form of the Ward identity takes the form

$\left(\left(\mathrm{D}_{\theta} \mathscr{A}_{\bar{\theta} a}\right)-\frac{\pi}{k} \mathrm{D}_{\bar{\theta}} \frac{\delta}{\delta \mathscr{A}_{\bar{\theta}}^{a}}-\frac{\mathrm{i} \pi}{k} f_{a b}{ }^{c} \mathscr{A}_{\bar{\theta}}^{b} \frac{\delta}{\delta \mathscr{A}_{\bar{\theta}}^{c}}\right) Z_{+}\left[\mathscr{A}_{\bar{\theta}}\right]=0$,

where $f_{a b c}$ are the structure constants of the group G. Eq. (9) yields a corresponding identity for the antiholomorphic superconformal block. In section 3, we attempt to reproduce these identities from a 3D viewpoint.

\section{Reproducing the superconformal blocks from 3D}

In order to reproduce eq. (10) for the superconformal blocks as a condition on the wavefunctions of a canonically quantized $3 \mathrm{D}$ theory, we follow the approach of refs. $[16,17]$. It will be shown in this section that the appropriate $3 \mathrm{D}$ action is

$S_{\mathrm{SCS}}^{+}[\mathscr{A}]=\frac{k}{2 \pi} \int \mathrm{d} t \int \mathrm{d} z \wedge \mathrm{d} \bar{z} \int \mathrm{d}^{2} \theta\left[\left(\mathscr{A}_{\theta}, \partial_{t} \mathscr{A}_{\bar{\theta}}\right)-\left(\mathscr{A}_{t}, \mathscr{F}_{\theta \bar{\theta}}\right)\right]$.

Here, the $2 \mathrm{D}$ superfields $\mathscr{A}_{\theta}$ and $\mathscr{A}_{\hat{\theta}}$ depend on the "time" coordinate $t$ on the $3 \mathrm{D}$ manifold $\Sigma \times \mathbb{R}, \mathscr{A}_{t}(t, Z, \bar{Z})$ is an unconstrained superfield and

$\mathscr{\mathscr { F }}_{\theta \bar{\theta}}=\mathrm{D}_{\theta} \mathscr{A}_{\bar{\theta}}+\mathrm{D}_{\bar{\theta}} \mathscr{A}_{\theta}+\left\{\mathscr{A}_{\theta}, \mathscr{A}_{\bar{\theta}}\right\}$.

Written in terms of component fields (see later), this action contains (up to a total derivative) the usual 3D bosonic CS action, although it must be stressed that $S_{\mathrm{SCS}}^{+}$does not possess 3D supersymmetry. It is only supersymmetric with respect to supersymmetry transformations on the "spacelike hypersurface" $\Sigma$, and $\theta$ and $\bar{\theta}$ are chiral spinors on $\Sigma$. This is similar to the corresponding situation for the topological term in the SWZW action.

Under a finite gauge transformation $\mathscr{G}(t, Z, \bar{Z}) \in \mathrm{G}$ with $\mathscr{A}_{\theta}^{\mathscr{G}}$ and $\mathscr{A}_{\theta}^{\mathscr{G}}$ defined as before and $\mathscr{A}_{t}^{\mathscr{G}}=\mathscr{G}^{-1}\left(\mathscr{A}_{t}+\partial_{t}\right) \mathscr{G}$, the $3 \mathrm{D}$ action is not invariant but transforms as

$$
\begin{aligned}
& S_{\mathrm{SCS}}^{+}\left[\mathscr{A}^{\mathscr{G}}\right]=S_{\mathrm{SCS}}^{+}[\mathscr{A}]+S_{\mathrm{SCS}}^{+}\left[\mathscr{G}^{-1} \mathrm{D} \mathscr{G}\right] \\
& \quad+\frac{k}{2 \pi} \int \mathrm{d} t \int \mathrm{d} z \wedge \mathrm{d} \bar{z} \int \mathrm{d}^{2} \theta\left[-\mathrm{D}_{\bar{\theta}}\left(\mathscr{A}_{\theta}, \partial_{t} \mathscr{G} \cdot \mathscr{G}^{-1}\right)-\mathrm{D}_{\theta}\left(\mathscr{A}_{\bar{\theta}}, \partial_{t} \mathscr{G} \cdot \mathscr{G}^{-1}\right)+\partial_{t}\left(\mathrm{D}_{\theta} \mathscr{G} \cdot \mathscr{G}^{-1}, \mathscr{A}_{\bar{\theta}}\right)\right],
\end{aligned}
$$

where $\mathscr{A}=\mathscr{G}^{-1} \mathrm{D} \mathscr{G}$ is a shorthand for

$\left(\mathscr{A}_{\theta}, \mathscr{A}_{\bar{\theta}}, \mathscr{A}_{t}\right)=\left(\mathscr{G}^{-1} \mathrm{D}_{\theta} \mathscr{G}, \mathscr{G}^{-1} \mathrm{D}_{\bar{\theta}} \mathscr{G}, \mathscr{G}^{-1} \partial_{t} \mathscr{G}\right)$.

The last term in (13) is a total derivative and vanishes if $\Sigma$ has no boundary and the gauge transformation vanishes at $\pm \infty$. It is easily verified that

$$
\begin{aligned}
& S_{\mathrm{SCS}}^{+}\left[\mathscr{G}^{-1} \mathrm{D} \mathscr{G}\right]=\frac{k}{4 \pi} \int \mathrm{d} t \int \mathrm{d} z \wedge \mathrm{d} \bar{z} \int \mathrm{d}^{2} \theta\left[-\mathrm{D}_{\bar{\theta}}\left(\mathscr{G}^{-1} \mathrm{D}_{\theta} \mathscr{G}, \mathscr{G}^{-1} \partial_{t} \mathscr{G}\right)-\mathrm{D}_{\theta}\left(\mathscr{G}^{-1} \mathrm{D}_{\bar{\theta}} \mathscr{G}, \mathscr{G}^{-1} \partial_{t} \mathscr{G}\right)\right. \\
& \left.\quad+\partial_{t}\left(\mathscr{G}^{-1} \mathrm{D}_{\theta} \mathscr{G}, \mathscr{G}^{-1} \mathrm{D}_{\bar{\theta}} \mathscr{G}\right)+\left(\mathscr{G}^{-1} \partial_{t} \mathscr{G},\left\{\mathscr{G}^{-1} D_{\theta} \mathscr{G}, \mathscr{G}^{-1} \mathrm{D}_{\bar{\theta}} \mathscr{G}\right\}\right)\right]
\end{aligned}
$$


By comparison with the topological term in the SWZW action (1), the last term in (14) is seen to be proportional to the winding number of the bosonic configuration given by the $\theta=0=\bar{\theta}$ component of $\mathscr{G}$, which vanishes on $\Sigma \times \mathbb{R}$. Thus the action is gauge invariant for gauge transformations vanishing at $t= \pm \infty$. This relation of the gauge variation of $S_{\mathrm{SCS}}^{+}$to the winding number of the gauge configuration will reproduce the quantization condition on $k$ from the SWZW theory if the functional integral which contains $\exp \left(\mathrm{i} S_{\mathrm{SCS}}^{+}\right)$is required to be well defined on more complicated three-manifolds which are locally of the form $\Sigma \times \mathbb{R}$.

The gauge freedom can be used to set $\mathscr{A}_{t}=0$. In this gauge, the action describes a set of free fields,

$S_{\mathrm{SCS}}^{+}[\mathscr{A}]=\frac{k}{2 \pi} \int \mathrm{d} t \int \mathrm{d} z \wedge \mathrm{d} \bar{z} \int \mathrm{d}^{2} \theta\left(\mathscr{A}_{\theta}, \partial_{\mathrm{t}} \mathscr{A}_{\bar{\theta}}\right)$,

whose canonical quantization yields the superfield anticommutation relations

$\left\{\mathscr{A}_{\theta}^{a}(Z, \bar{Z}), \mathscr{A}_{\theta}^{b}(W, \bar{W})\right\}=-\frac{\pi}{k} g^{a b} \delta^{(2)}(z-w) \delta^{(2)}\left(\theta-\theta^{\prime}\right)$

where $g_{a b}=\left(T_{a}, T_{b}\right)$ for generators $T_{a}$ of $\mathrm{g}$. This can be verified by writing the action out in components, remembering $\delta^{(2)}\left(\theta-\theta^{\prime}\right)=\left(\bar{\theta}-\bar{\theta}^{\prime}\right)\left(\theta-\theta^{\prime}\right)$. Since only $\mathscr{A}_{\bar{\theta}}$ appears in the action with a time derivative, the components of this superfield play the role of canonical coordinates, and the wavefunction $\Psi$ is a functional of the component fields of $\mathscr{A}_{\bar{\theta}}$, denoted $\Psi=\Psi\left[\mathscr{A}_{\bar{\sigma}}\right]$. The canonical anticommutation relations are realized on the wavefunctions via the identification

$\mathscr{A}_{\theta}^{a}(Z, \bar{Z})=-\frac{\pi}{k} g^{a b} \frac{\delta}{\delta \mathscr{A}_{\theta}^{b}(Z, \bar{Z})}$.

The $\mathrm{n}^{r}$ - linearity of the theory (as in ref. [1]) is contained in the equation of motion $\mathscr{\mathscr { F }}_{\theta \bar{\theta}}=0$ for $\mathscr{A}_{t}$, which contains no time derivatives and so is an equation of constraint. The superfield $\mathscr{\mathscr { F }}_{\theta \theta}$ acts as the generator of infinitesimal time-independent gauge transformations of $\mathscr{A}_{\theta}$ and $\mathscr{A}_{\theta}$ via the canonical anticommutation relations, so the constraint is first class. In the quantum theory, the constraint equation is realized on the space of wavefunctions as

$\mathscr{F}_{\theta \bar{\theta}} \Psi\left[\mathscr{A}_{\theta}\right]=0$

which, using (16), is the equation

$\left(\left(\mathrm{D}_{\theta} \mathscr{A}_{\bar{\theta} a}\right)+\frac{\pi}{k} \mathrm{D}_{\bar{\theta}} \frac{\delta}{\delta \mathscr{A}_{\bar{\theta}}^{a}}-\frac{\mathrm{i} \pi}{k} f_{a b} \mathscr{A}^{b} \frac{\delta}{\delta \mathscr{A}_{\bar{\theta}}^{c}}\right) \Psi\left[\mathscr{A}_{\bar{\theta}}\right]=0$.

This is equivalent to the anomalous Ward identity (10) if we identify the holomorphic superconformal block $Z_{+}\left[\mathscr{A}_{\bar{\theta}}\right]$ with the wavefunction $\Psi\left[\mathscr{A}_{\bar{\theta}}\right]$, the promised result.

The behaviour of the wavefunction under finite gauge transformations is most easily obtained by considering $\Psi\left[\mathscr{A}_{\theta}\right]$ as the result of performing the functional integral $\int[\mathrm{d} \mathscr{A}] \exp \left(\mathrm{i} S_{\mathrm{SCS}}^{+}[\mathscr{A}]\right)$ on $\Sigma \times \mathbb{R}^{+}$with $\mathscr{A}_{\theta}$ prescribed on the boundary surface $t=0$. Using (13) and (14), it follows that

$\Psi[\mathscr{A} \mathscr{\theta}]=\exp \left(S_{\mathrm{WZW}}\left[\mathscr{G}, \mathscr{A}_{\theta}=0, \mathscr{A}_{\theta}\right]\right) \Psi\left[\mathscr{A}_{\theta}\right]$

The action $S_{\mathrm{SCS}}^{+}[\mathscr{A}]$ is in fact one of a large class characterized by the equations of motion

$\frac{\delta S}{\delta \mathscr{A}_{\theta}}=-\frac{\mathrm{i} k}{\pi} \mathscr{F}_{1 \bar{\theta}}, \quad \frac{\delta S}{\delta \mathscr{A}_{\bar{\theta}}}=-\frac{\mathrm{i} k}{\pi} \mathscr{F}_{t \theta}, \quad \frac{\delta S}{\delta \mathscr{A}_{t}}=\frac{\mathrm{i} k}{\pi} \mathscr{F}_{\theta \bar{\theta}}$

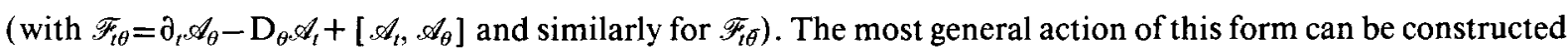


by integrating these equations in the same manner that one constructs Wess-Zumino effective actions [18] and differs from (11) by total derivatives. Gauge invariance of the actions (with appropriate boundary conditions on the gauge transformations) results from the Bianchi identity $\mathscr{D}_{\theta} \mathscr{F}_{t \theta}+\mathscr{D}_{\theta} \mathscr{F}_{1 \bar{\theta}}-\mathscr{D}_{t} \mathscr{\mathscr { F }}_{\theta \bar{\theta}}=0$, where $\mathscr{D}$ denotes gauge covariant derivatives. Of particular interest are the actions

$S_{\overline{\mathrm{SCS}}}[\mathscr{A}]=\frac{k}{2 \pi} \int \mathrm{d} t \int \mathrm{d} z \wedge \mathrm{d} \bar{z} \int \mathrm{d}^{2} \theta\left[\left(\mathscr{A}_{\bar{\theta}}, \partial_{t} \mathscr{A}_{\theta}\right)-\left(\mathscr{A}_{t}, \mathscr{\mathcal { F }}_{\theta \bar{\theta}}\right)\right]$,

whose canonical quantization reproduces the antiholomorphic superconformal blocks $Z_{-}\left[\mathscr{A}_{\theta}\right]$ and the symmetric version

$S_{\mathrm{SCS}}[\mathscr{A}]=\frac{k}{2 \pi} \int \mathrm{d} t \int \mathrm{d} z \wedge \mathrm{d} \bar{z} \int \mathrm{d}^{2} \theta\left[\frac{1}{2}\left(\mathscr{A}_{\theta}, \mathscr{F}_{t \theta}\right)+\frac{1}{2}\left(\mathscr{A}_{\bar{\theta}}, \mathscr{\mathscr { H }}_{\theta}\right)-\frac{1}{2}\left(\mathscr{A}_{t}, \mathscr{\mathscr { F }}_{\theta \theta}\right)+\frac{1}{2}\left(\mathscr{A}_{t},\left\{\mathscr{A}_{\theta}, \mathscr{A}_{\hat{\theta}}\right\}\right)\right]$,

which contains the usual bosonic CS action in its component expansion.

\section{The reduced phase space}

In the previous section, the $3 \mathrm{D}$ theory with action $S_{\mathrm{SCS}}^{+}[\mathscr{A}]$ was quantized on the phase space of all superconnections $\left\{\mathscr{A}_{\theta}, \mathscr{A}_{\bar{\theta}}\right\}$ and then the constraint $\mathscr{F}_{\hat{\theta}}=0$ was imposed on the wavefunctions. According to the general principles of geometric quantization (see, for example, ref. [19]) this is equivalent to the statement the physical wavefunctions are defined on the reduced phase space of superconnections $\left\{\mathscr{A}_{\theta}, \mathscr{A}_{\hat{\theta}}\right\}$ with vanishing curvature modulo the action of the group of gauge transformations taking values in $G$. In this section, we will examine the structure of the reduced phase space. Attention is confined to the case where the superconformal structure on $\Sigma$ is "split", meaning that there is no nontrivial gravitino background (i.e., the gravitino background is pure gauge). In this case, $\theta$ is an ordinary chiral spinor on $\Sigma$ in a given spin structure [8]. The nonsplit case is discussed in the conclusion.

We will first show that the constraint $\tilde{\mathscr{T}}_{\theta \bar{\theta}}=0$ means that $\mathscr{A}_{\theta}$ and $\mathscr{A}_{\bar{\theta}}$ are equivalent to the superfields $\tilde{\mathscr{A}}_{\theta}$ and $\tilde{\mathscr{A}}_{\bar{\theta}}$ which have the particularly simple form

$\tilde{\mathscr{A}}_{\theta}=\theta \tilde{\mathscr{A}}_{z}=\theta\left(A_{z}+\frac{1}{2}\left\{A_{\theta}, A_{\theta}\right\}\right), \quad \tilde{\mathscr{A}}_{\bar{\theta}}=\bar{\theta} \tilde{\mathscr{A}}_{\bar{z}}=\bar{\theta}\left(A_{\bar{z}}+\frac{1}{2}\left\{A_{\bar{\theta}}, A_{\bar{\theta}}\right\}\right)$,

with $\tilde{F}_{z \bar{z}}=\partial_{z} \tilde{A}_{\bar{z}}-\partial_{\bar{z}} \tilde{A}_{z}+\left[\tilde{A}_{z}, \tilde{A}_{\bar{z}}\right]=0$. To show this, one requires the transformation properties of $\mathscr{A}_{\theta}$ and $\mathscr{A}_{\tilde{\theta}}$ under finite gauge transformations. If $\mathscr{A}_{\theta}$ and $\mathscr{A}_{\bar{\theta}}$ have the component expansions

$\mathscr{A}_{\theta}=A_{\theta}+\theta A_{z}+\bar{\theta} A_{\bar{\theta} \theta}+\bar{\theta} \theta A_{z \bar{\theta}}, \quad \mathscr{A}_{\bar{\theta}}=A_{\bar{\theta}}+\theta A_{\theta \theta}+\bar{\theta} A_{\bar{z}}+\bar{\theta} \theta A_{\bar{z} \theta}$,

then under a finite gauge transformation by $\mathscr{G}=\exp (\mathrm{i} \Lambda)=\exp \left[\mathrm{i}\left(\theta \lambda_{\theta}+\bar{\theta} \lambda_{\theta}+\bar{\theta} \theta \lambda_{\bar{\theta} \theta}\right)\right]$ with $A \in \mathrm{g}$ [this is not the most general allowed gauge transformation, which is of the form $g \exp (\mathrm{i} \Lambda)]$,

$$
\begin{aligned}
& \mathscr{A}_{\theta}^{\prime \prime}=A_{\theta}+\mathrm{i} \lambda_{\theta}+\theta\left(A_{z}-\mathrm{i}\left\{\lambda_{\theta}, A_{\theta}\right\}+\frac{1}{2}\left\{\lambda_{\theta}, \lambda_{\theta}\right\}\right)+\bar{\theta}\left(A_{\bar{\theta} \theta}-\mathrm{i} \lambda_{\bar{\theta} \theta}-\mathrm{i}\left\{\lambda_{\bar{\theta}}, A_{\theta}\right\}+\frac{1}{2}\left\{\lambda_{\theta}, \lambda_{\bar{\theta}}\right\}\right) \\
& \quad+\bar{\theta} \theta\left(A_{\bar{z} \bar{\theta}}-\mathrm{i} \mathrm{D}_{\bar{z}} \lambda_{\bar{\theta}}-\mathrm{i}\left[\lambda_{\theta}, A_{\bar{\theta} \theta}\right]-\mathrm{i}\left[\lambda_{\bar{\theta} \theta}, A_{\theta}\right]-\frac{1}{2}\left[\lambda_{\theta},\left\{\lambda_{\bar{\theta}}, A_{\theta}\right\}\right]\right. \\
& \left.+\frac{1}{2}\left[\lambda_{\bar{\theta}},\left\{A_{\theta}, \lambda_{\theta}\right\}\right]-\left[\lambda_{\theta}, \lambda_{\bar{\theta} \theta}\right]+\frac{1}{4} \mathrm{i}\left[\left\{\lambda_{\theta}, \lambda_{\theta}\right\}, \lambda_{\bar{\theta}}\right]\right), \\
& \mathscr{A}_{\bar{\theta}}^{\mathscr{G}}=A_{\bar{\theta}}+\mathrm{i} \lambda_{\bar{\theta}}+\theta\left(A_{\theta \bar{\theta}}+\mathrm{i} \lambda_{\bar{\theta} \theta}-\mathrm{i}\left\{\lambda_{\theta}, A_{\bar{\theta}}\right\}+\frac{1}{2}\left\{\lambda_{\theta}, \lambda_{\bar{\theta}}\right\}\right)+\bar{\theta}\left(A_{\bar{z}}-\mathrm{i}\left\{\lambda_{\bar{\theta}}, A_{\bar{\theta}}\right\}+\frac{1}{2}\left\{\lambda_{\bar{\theta}}, \lambda_{\bar{\theta}\}}\right\}\right. \\
& \quad+\bar{\theta} \theta\left(A_{\bar{z} \theta}+\mathrm{i} \mathrm{D}_{\bar{z}} \lambda_{\theta}+\mathrm{i}\left[\lambda_{\bar{\theta}}, A_{\theta \bar{\theta}}\right]-\mathrm{i}\left[\lambda_{\bar{\theta}}, A_{\bar{\theta}}\right]-\frac{1}{2}\left[\lambda_{\theta},\left\{\lambda_{\bar{\theta}}, A_{\bar{\theta}}\right\}\right]\right. \\
& \left.\quad+\frac{1}{2}\left[\lambda_{\bar{\theta}},\left\{\lambda_{\theta}, A_{\bar{\theta}}\right\}\right]-\left[\lambda_{\bar{\theta}}, \lambda_{\bar{\theta} \theta}\right]+\frac{1}{4} \mathrm{i}\left[\left\{\lambda_{\bar{\theta}}, \lambda_{\theta}\right\}, \lambda_{\theta}\right]\right),
\end{aligned}
$$

where $\mathrm{D}_{z} \lambda_{\bar{\theta}}=\partial_{z} \lambda_{\bar{\theta}}+\left[A_{z}, \lambda_{\theta}\right]$

The superfield constraint $\mathscr{F}_{\theta \theta}=0$ is equivalent to the following constraint equations for the component fields: 
$A_{\theta \bar{\theta}}+A_{\bar{\theta} \theta}+\left\{A_{\theta}, A_{\bar{\theta}}\right\}=0, \quad A_{z \bar{\theta}}=-\tilde{\mathrm{D}}_{z} A_{\bar{\theta}}-\left[A_{\theta}, A_{\bar{\theta} \theta}\right], \quad A_{\bar{z} \theta}=\tilde{\mathrm{D}}_{\bar{z}} A_{\theta}+\left[A_{\bar{\theta}}, A_{\theta \theta}\right]$,

$\tilde{F}_{z \bar{z}}=0$.

By choosing $\mathrm{i} \lambda_{\theta}=-A_{\theta}, \mathrm{i} \lambda_{\bar{\theta}}=-A_{\bar{\theta}}$ and $\mathrm{i} \lambda_{\bar{\theta}}=A_{\bar{\theta} \theta}+\frac{1}{2}\left\{A_{\theta}, A_{\bar{\theta}}\right\}$ and making use of the constraint equations (22), (23) and the Jacobi identities, (20) and (21) reduce to

$\mathscr{A}_{\theta}^{\mathscr{\theta}}=\theta \tilde{A}_{z}, \quad \mathscr{A}_{\bar{\theta}}^{\mathscr{G}}=\bar{\theta} \tilde{A}_{\bar{z}}$.

So, the result is that superfields $\mathscr{A}_{\theta}, \mathscr{A}_{\bar{\theta}}$ obeying the constraint $\mathscr{F}_{\theta \bar{\theta}}=0$ are gauge equivalent to the flat [via (23)] bosonic connection $\left(\tilde{A}_{z}, \tilde{A}_{z}\right)$. As pointed out, the full gauge group is not used in achieving this result, there still remains the freedom to make purely bosonic gauge transformations with $\mathscr{G}=g(z, \bar{z})$. Thus the reduced phase space is equivalent to the space of flat bosonic connections on $\Sigma$ modulo the action of the group of bosonic gauge tranformations taking values in G. This is a satisfactory state of affairs, since, as emphasized in ref. [1], this space is canonically associated with the surface $\Sigma$ independent of any choice of metric (and hence complex structure ) or spin structure. In the case of ordinary conformal blocks, the canonical nature of the reduced phase space allows the conformal blocks associated with different points on Teichmüller space to be canonically identified and can be interpreted as the presence of a projectively flat connection over moduli space in the spirit of the program of modular geometry of Friedan and Shenker [20]. As we shall point out, in the superconformal case the superconformal blocks are associated with an additional structure over this space of flat bosonic connections, but the canonical structure of this space offers the hope of identifying the supersymmetric analogue of the projectively flat connection over moduli space.

In order to identify the additional structure referred to in the above paragraph, it is useful to examine how the wavefunction $\Psi\left[\mathscr{A}_{\theta}\right]$ defined on the space of all $\left\{\mathscr{A}_{\theta}, \mathscr{A}_{\hat{\theta}}\right\}$ reduces to a physical wavefunction defined on the reduced phase space. This is achieved by attempting to express $\mathscr{A}_{\bar{\theta}}$ in the form $\mathscr{G}^{-1} \mathrm{D}_{\bar{\theta}} \mathscr{G}$ (or, equivalently, $\mathscr{A}_{\bar{\theta}}^{\mathscr{O}}=0$ ) with $\mathscr{G}$ a globally well-defined superfield on $\Sigma$ taking values in $\mathrm{G}^{\mathbb{C}}$. (Note that this is not equivalent to the superconnection being pure gauge; this is only the case when $\mathscr{G}$ takes values in $\mathrm{G}$, as then with $\mathscr{A}_{\theta}=-\mathscr{A}_{\theta}^{*}$ the curvature $\mathscr{F}_{\theta \bar{\theta}}$ vanishes.) There is an obstruction to writing $\mathscr{A}_{\bar{\theta}}$ in this form which is related to the additional structure on the reduced phase space.

We make the reduction in two steps by writing $\mathscr{G}=\mathscr{G}_{2} \mathscr{G}_{1}$, and choosing $\mathscr{G}_{1}=\exp \left[-\mathrm{i}\left(\bar{\theta} \lambda_{\bar{\theta}}+\bar{\theta} \theta \lambda_{\bar{\theta} \theta}\right)\right]$ with $\mathrm{i} \lambda_{\bar{\theta}}=-A_{\bar{\theta}}, \mathrm{i} \lambda_{\bar{\theta} \theta}=-A_{\theta \bar{\theta}}$. Then using (21),

$\mathscr{A}_{\bar{\theta}}^{\mathscr{\theta}^{-1}}=\tilde{\theta} \tilde{A}_{\bar{z}}+\bar{\theta} \theta\left(A_{\bar{z} \theta}+\left[A_{\theta \dot{\theta}}, A_{\bar{\theta}}\right]\right)$.

Since $A_{\bar{\theta}}$ and $A_{\theta \theta}$ are globally defined spinors (which follows from the fact that $\mathscr{A}_{\bar{\theta}}$ is a globally defined spinor superfield), $\mathscr{G}_{1}$ is also a globally well-defined mapping from $\Sigma$ into $G^{\mathrm{C}}$. However, we will find that there is an obstruction to writing $\mathscr{A}_{\bar{\theta}} \Gamma^{-1}$ in the form $\mathscr{G}_{2}^{-1} \mathrm{D}_{\bar{\theta}} \mathscr{G}_{2}$.

We choose $\mathscr{G}_{2}=g \exp \left(\mathrm{i} \theta \lambda_{\theta}\right)$ with $g(z, \bar{z}) \in \mathrm{G}^{\mathbb{C}}$. Equating $\mathscr{A}_{\theta}^{\mathscr{H}^{-1}}$ and $\mathscr{G}_{2}^{-1} \mathrm{D}_{\bar{\theta}} \mathscr{G}_{2}$, one finds

$\tilde{A}_{z}=g^{-1} \partial_{z} g$,

$A_{\bar{z} \theta}+\left[A_{\theta \bar{\theta}}, A_{\bar{\theta}}\right]=\mathrm{i}\left(\partial_{\bar{z}} \lambda_{\theta}+\left[g^{-1} \partial_{\bar{z}} g, \lambda_{\theta}\right]\right)$.

Turning first to (24), there is an obstruction to expressing the (nonflat) connection $\tilde{A}_{\bar{z}}$ in this form with $g$ a globally well-defined map from $\Sigma$ into $\mathrm{G}^{\mathrm{C}}$. However, within the $\mathrm{G}^{\mathrm{C}}$ gauge orbit of $A_{\bar{z}}$ (with $\tilde{A}_{z}=-\tilde{A}_{\bar{z}}^{*}$ ), there is a flat connection $[21,14]$. Thus we can write $\tilde{A}_{\bar{z}}=A_{\bar{z}}(u)^{g}$, where $g$ is globally well defined and $A_{\bar{z}}(u)$ is a flat connection parameterized by (complex) coordinates $u$ on the space of flat connections on $\Sigma$ (these coordinates are related to the holonomies of the flat connection, see refs. [22,14]). Actually, since $G \subset G^{C}$, the $G^{C}$ gauge 
orbit is characterized by a point in the moduli space of flat connections modulo gauge transformations taking values in $\mathrm{G}$.

Eq. (25) can now be reexpressed $A_{z \theta}^{\prime}=\mathrm{iD} \mathrm{D}_{z}(u) \lambda_{\theta}^{\prime}$, where $A_{\bar{z} \theta}^{\prime}$ and $\lambda_{\theta}^{\prime}$ are suitably redefined quantities with $A_{\bar{z} \theta}^{\prime}$ a globally defined vector-spinor, and $\mathrm{D}_{\bar{z}}(u)$ the covariant derivative with respect to the flat connection $A_{\bar{z}}(u)$. Again, there is in general an obstruction to fulfilling this equation for a globally well-defined spinor which is classified by the equivalence classes $\left[A_{\bar{z} \theta}^{\prime}\right]$ of tensors under the equivalence relation $A_{\bar{z} \theta}^{\prime} \sim A_{z \theta}^{\prime}+\mathrm{D}_{\bar{z}}(u) \lambda_{\theta}^{\prime}$ with $\lambda_{\theta}^{\prime}$ a globally well-defined spinor. The equivalence classes $\left[A_{\bar{z} \theta}^{\prime}\right]$ form a vector space $\mathrm{V}(u)$ whose dimension can be established by index theory; it is finite dimensional.

To summarize, it has been shown that a point in $\left\{\mathscr{A}_{\theta}, \mathscr{A}_{\theta}\right\} / \mathscr{G}^{\mathrm{C}}$ is equivalent to a point $u$ on the moduli space of flat bosonic connections modulo G-valued gauge transformations plus a vector $\xi(u)$ in the vector space $\mathrm{V}(u)$ of equivalence classes $\left[A_{z \theta}^{\prime}\right]$ over this point. The latter is the extra structure on the reduced phase space in the superconformal case alluded to earlier. The equivalence classes $\left[A_{z \theta}^{\prime}\right]$ are supermoduli.

Using the above results and (18), it is thus possible to reduce the wavefunction $\Psi\left[\mathscr{A}_{\theta}\right]$ in the form

$\Psi\left[\mathscr{A}_{\theta}\right]=\exp \left(S_{\mathrm{wZW}}\left[\mathscr{G}, \mathscr{A}_{\theta}, \mathscr{A}_{\hat{\theta}}\right]\right) \Psi[u, \xi(u)]$,

with $\mathscr{G}$ an appropriately chosen $\mathrm{G}^{\mathbb{C}}$-valued gauge transformation and where $\Psi[u, \xi(u)]$ is the physical wavefunction on the reduced phase space. If one chooses a basis for the vector space $V(u)$, then the vector $\xi(u)$ is characterized by Grassmann-valued parameters $\xi^{i}(u)$ relative to this basis, and the wavefunction can be expanded in the Grassmann parameters as

$\Psi[u, \xi(u)]=\psi_{0}(u)+\xi^{i}(u) \psi_{i}(u)+\xi^{i}(u) \wedge \xi^{j}(u) \psi_{i j}(u)+\ldots$.

The components $\psi_{i j . .}(u)$ take values in the wedge product of appropriate order of $\mathrm{V}(u)$ with itself. With the notation $S_{\mathrm{WZW}}\left[\mathscr{G}, \mathscr{A}_{\theta}=0, \mathscr{A}_{\bar{\theta}}\right] \equiv \mathrm{S}[\mathscr{G}, \mathscr{A}]$, the exponent in (26) obeys the cocycle condition

$0=S\left[\mathscr{G}_{1}, \mathscr{A}\right]+S\left[\mathscr{G}_{2}, \mathscr{A}^{\mathscr{C}_{1}}\right]+S\left[\left(\mathscr{G}_{1} \mathscr{G}_{2}\right)^{-1}, \mathscr{A}^{\mathscr{S}_{1} \mathscr{\mathscr { S }}_{2}}\right]$,

as can be verified using (3) and (4). This ensures that $\Psi\left[\mathscr{A}_{\bar{\theta}}\right]$ reduces consistently to a section of a vector bundle over the reduced phase space. This is of the form $L \otimes\left(\oplus_{i} \wedge^{i} \mathrm{E}\right)$, where $\mathrm{L}$ is the line bundle in which $\psi_{0}(u)$ takes values and $\mathrm{E}$ is the vector bundle with fiber $\mathrm{V}(u)$ over $u$. This is the usual form of the wavefunction for supersymmetric systems (see, for example, ref. [23]).

In the next section, we illustrate this structure for the case when the split super Riemann surface $\Sigma$ is the torus with the $(++)$-spin structure for the fermions.

\section{The torus case}

Flat bosonic connections on the torus are characterized by their holonomies $g_{\alpha}$ and $z \rightarrow g_{\beta}$ around the $\alpha$ and $\beta$ cycles (corresponding to $z \rightarrow z+1$ and $z \rightarrow z+\tau$ respectively, where $\tau$ is the modular parameter on the torus). Due to the relation $g_{\alpha} g_{\beta} g_{\alpha}^{-1} g_{\beta}^{-1}=1$ and the equivalence of connections with holonomies related by conjugation by a constant element in $\mathrm{G}, g_{\alpha}$ and $g_{\beta}$ can be chosen to take values in the maximal torus T of G [14,22]. If we write $g_{\alpha}=\exp (-2 \pi \mathrm{i} \Phi)$ and $g_{\beta}=\exp (-2 \pi \mathrm{i} \Theta)$ with $\Phi, \Theta \in \mathrm{t}$, the Cartan subalgebra of $\mathrm{g}$, then $\Theta+M, \Phi-N$ with $M$ and $N$ coroot vectors $[M, N \in \mathrm{t}$ and $\exp (2 \pi \mathrm{i} M)=1=\exp (2 \pi \mathrm{i} N)]$ determine the same holonomies. Also, there remains the freedom to make a simultaneous Weyl transformation of $\Theta$ and $\Phi$, which is what survives of the equivalence of holonomies under conjugation after choosing $g_{\alpha}$ to lie in $\mathrm{T}$. The flat connections on the torus can thus be parameterized by $u=\Theta-\tau \Phi$ with the identifications $u \sim u+M+N \tau$ and $u \sim w(u)$, where $w$ denotes an element of the Weyl group. A particular realization is given by

$A_{z}(u)=g(u, \bar{u})^{-1} \partial_{\bar{z}} g(u, \bar{u}), \quad A_{z}(\bar{u})=-A_{z}(u)^{*}$,

with $g(u, \bar{u})=\exp \{[2 \pi \mathrm{i} /(\tau-\bar{\tau})](u \bar{z}-\bar{u} z)\}$. 
Over a point $u$ on the moduli space of flat bosonic connections, we will examine the vector space of equivalence classes $\left[A_{z \theta}\right]$ under the equivalence relation $A_{\bar{z} \theta} \sim A_{\bar{z} \theta}+\mathrm{D}_{\bar{z}}(u) \lambda_{\theta}$ for $\lambda_{\theta}$ a well-defined spinor taking values in $\mathrm{g}^{\mathbb{C}}$, and $\mathrm{D}_{z}(u) \lambda_{\theta}=\partial_{z} \lambda_{\theta}+\left[A_{z}(u), \lambda_{\theta}\right]$. This is dual to the vector space of zero modes $A_{\theta}$ of the operator $\mathrm{D}_{\bar{z}}(u)$ via the inner product $\frac{1}{2} \mathrm{i} \int \mathrm{d} z \wedge \mathrm{d} \bar{z}\left(A_{\theta}, A_{\bar{z} \theta}\right)$. Thus it suffices to find the kernel of $\mathrm{D}_{\bar{z}}(u)$. Using $(28)$, $\mathrm{D}_{\bar{z}}(u) A_{\theta}=0$ is equivalent to

$\partial_{\bar{z}}\left[g(u, \bar{u}) A_{\theta} g(u, \bar{u})^{-1}\right]=0$.

For the $(++)$-spin structure, the spin bundle is trivial and so $A_{\theta}$ transforms by the transition function 1 around $\alpha$ and $\beta$ cycles. Decomposing $A_{\theta}=A_{\theta}^{\alpha} E_{\alpha}+A_{\theta}^{-\alpha} E_{-\alpha}+A_{\theta}^{i} H_{i}$, where $E_{\alpha}$ are the generators of $g$ associated with the positive roots and $H_{i}$ is a basis for the Cartan subalgebra, one finds that $g(u, \bar{u}) A_{\bar{\theta}}^{ \pm a} g(u, \bar{u})^{-1}$ are sections of flat line bundles over $\Sigma$ with transition functions $\exp [\mp 2 \pi \mathrm{i} \alpha(\Phi)]$ and $\exp [\mp 2 \pi \mathrm{i} \alpha(\Theta)]$ around the $\alpha$ and $\beta$ cycles respectively, while $g(u, \bar{u}) A_{\theta}^{i} g(u, \bar{u})^{-1}$ are sections of the trivial line bundle. Using (29), $\operatorname{ker} \mathrm{D}_{\bar{z}}(u)$ is equivalent to the space of holomorphic sections of these flat line bundles. If one excludes the connections $A_{z}(u)$ corresponding to $\Phi$ and $\Theta$ being coroot vectors [14], there are no holomorphic sections of the line bundles associated with $A_{\theta}^{ \pm \alpha}$, while for each $A_{\theta}^{i}$ there is a one-dimensional space of holomorphic sections, namely the constant sections. So we find that the vector space of equivalence classes $\left[A_{z \theta}\right]$ has dimension rank $\mathrm{G}$, which is equal to the dimension of the moduli space of flat connections. This is a reflection of the rigid supersymmetry due to the presence of a conformal Killing spinor on the torus with the $(++)$-spin structure, and the supermodular parameters parameterizing the classes $\left[A_{z \theta}\right]$ can be considered as superpartners of the modular parameters $u$. The nontrivial equivalence classes $\left[A_{z \theta}\right.$ ] associated with the modular parameter $u$ can be represented by $A_{z \theta}^{i}=$ $-\left[\xi^{i}(u)\right] /(\tau-\bar{\tau})$, where $\xi^{i}(u)$ is a constant (i.e., independent of $z$ and $\bar{z}$ ) Grassmann parameter, as these cannot be written in the form $\mathrm{D}_{\bar{z}}(u) \lambda_{\theta}^{i}$ for $\lambda_{\theta}^{i}$ with trivial transition functions on the torus. For the other spin structures on the torus, $A_{\bar{z} \theta}$ has nontrivial transition functions and these constant sections are ruled out, so there are no supermoduli.

To determine the nature of the vector bundle over the moduli space of flat connections given by the vector spaces $\mathrm{V}(u)$ of equivalence classes $\left[A_{z \theta}\right]$, it is necessary to determine the behaviour of $\xi^{i}(u)$ under the transformations $u \rightarrow u+M+N \tau, u \rightarrow w(u)$. These are equivalent to the gauge transformations $A_{\bar{z}}(u) \rightarrow A_{\bar{z}}(u)^{g M N}$ and $A_{\bar{z}}(u) \rightarrow A_{\bar{z}}(u)^{h-1}$ respectively, where $g_{M N}(z, \bar{z})=\exp \{[2 \pi \mathrm{i} /(\tau-\bar{\tau})][(M+N \tau) \bar{z}-(M+N \tau) z]\}$ and $w(u)=$ $h u h^{-1}$ for $h \in \mathrm{N}(\mathrm{T})$, the normalizer of the torus. Using the gauge transformation properties of the superfield $\mathscr{A}_{\hat{\theta}}$, it follows that $\xi^{i}(u+M+N \tau)=\xi^{i}(u)$, but $\xi^{i}(w(u)) H_{i}=w\left(\xi^{i}(u) H_{i}\right)$. Thus $\mathrm{V}(u+M+N \tau)$ and $\mathrm{V}(u)$ can be identified but $\mathrm{V}(w(u))$ differs from $\mathrm{V}(u)$ by the action of the Weyl group. As a result, the vector spaces $\mathrm{V}(u)$ determine a twisted vector bundle over the moduli space of flat connections.

The behaviour of the physical wavefunctions $\Psi[u, \xi(u)]$ under these gauge transformations follows from (18) for $\mathscr{G}=g_{M N}(z, \bar{z})$ and $\mathscr{G}=h$. One finds that $f[u, \xi(u)]=\exp \{[-\mathrm{i} \pi k /(\tau-\bar{\tau})](u, u)\} \Psi[u, \xi(u)]$ satisfies $f[u+M, \xi(u+M)]=f[u, \xi(u)]$,

$f[u+N \tau, \xi(u+N \tau)]=\exp [-\mathrm{i} \pi k \tau(N, N)-2 \pi \mathrm{i} k(N, u)] f[u, \xi(u)]$,

$f[w(u), \xi(w(u))]=f[u, \xi(u)]$.

Making use of the expansion (27) of $\Psi[u, \xi(u)]$, this means in particular that a basis for the top component $\xi^{1}(u) \wedge \xi^{2}(u) \wedge \ldots \wedge \xi^{r}(u) \psi_{12 \ldots r}(u)$ (with $r=$ rank $G$ ) is given by the expressions

$\exp \left[\left(\frac{\mathrm{i} \pi k}{\tau-\bar{\tau}}\right)(u, u)\right] \xi^{1}(u) \wedge \xi^{2}(u) \wedge \ldots \wedge \xi^{r}(u) \sum_{w \in \mathrm{W}}(-1)^{l(w)} \Theta_{w(\lambda), k}(\tau, u)$

where $\mathrm{W}$ denotes the Weyl group and $l(w)$ the parity of an element $w \in \mathrm{W}, \Theta_{\lambda, k}(\tau, u)$ is a level $k$ theta function, and $\lambda$ is a weight with the property $0 \leqslant(\lambda, \psi) \leqslant k$ (where $\psi$ is the highest root of $\mathrm{g}$ ). The presence of the level $k$ theta functions is due to the fact that they provide a basis for the multivalued functions on the moduli space of 
flat connections with the periodicity properties (30). The Weyl invariance condition (31) means that a linear combination of theta functions antisymmetric under the action of the Weyl group must appear, as the wedge product $\xi^{1}(u) \wedge \xi^{2}(u) \wedge \ldots \wedge \xi^{r}(u)$ is antisymmetric under the action of the Weyl group.

Precisely this structure arises when one considers the superconformal blocks for the level $k$ SWZW model on the torus with the $(++)$-spin structure in the presence of a background gauge field $A_{z}(u)$. The superconformal blocks of the gauged SWZW model on the torus are proportional to characters of super Kac-Moody (SKM) algebras. In the case of a split super Riemann surface (no nontrivial gravitino background), the character for a level $k$ SKM algebra factorizes into the product of an ordinary Kac-Moody character of level $k-g$ (where $g$ is the Coxeter number of $\mathrm{G}$ ) and a partition function for a set of free Weyl fermions $\psi_{\theta}$ in the adjoint representation [24]. If the $(++)$-spin structure is chosen on the torus, there are fermion zero modes in the presence of the background gauge field $A_{\bar{z}}(u)$ [the analogous of $\mathrm{D}_{\bar{z}}(u) A_{\theta}=0$ ] and the fermion partition function vanishes unless these are "soaked up" by appropriate insertions of fermion operators. These insertions arise via terms $\int \mathrm{d} z \wedge \mathrm{d} \bar{z}\left(A_{z \theta}, \psi_{\theta}\right)$ in the component expansion of the SWZW action (1) in the presence of background supergauge fields characterized by the nontrivial equivalence classes $\left[A_{z \theta}\right]$. The result $[25]$ is that up to the factor $\exp \{[\mathrm{i} \pi k /(\tau-\bar{\tau})](u, u)\}$, the SKM character is proportional to the expression in (32) (the difference between the results is due to the fact that the SKM character is computed using a connection $A_{z}(u)$ in which all the holonomy is around the $\beta$ cycle, as appropriate for the operator computation of two-dimensional chiral partition functions on the torus).

\section{Conclusion}

A $3 \mathrm{D}$ theory has been presented for which the wavefunctions resulting from the canonical quantization of the theory provide a realization of the 2D WZW superconformal blocks. The general structure of the Hilbert space of states has been examined in section 4 , and this structure has been illustrated in detail for the case of the torus with the $(++)$-spin structure in section 5 . We make the following additional remarks on the structure of the theory.

(i) So far, the dynamics of the 3D theory have not been considered. Since the action (11) is linear in time derivatives, the hamiltonian vanishes weakly (it is proportional to the generator $\mathscr{F}_{\theta \theta}$ of gauge transformations, and so vanishes on the space of physical states). Also, as has been discussed in section 4 , the space of states is finite dimensional, because the states are associated with a finite-dimensional vector bundle (related to an index problem ) over the finite-dimensional moduli space of flat bosonic connections. These facts suggest that the 3D theory described by the action (11) is a topological theory, as in the case of the ordinary Chern-Simons theory [1].

(ii) Although the space of states for the theory (11) has been examined, no discussion of an inner product on this space has been presented. By analogy with the bosonic case $[15,2,17]$, we suggest that the inner product on the space of states $\Psi\left[\mathscr{A}_{\theta}\right]$ is provided by

$$
\left\langle\Psi^{(1)} \mid \Psi^{(2)}\right\rangle=\int\left[\mathrm{d} \mathscr{A}_{\theta}\right]\left[\mathrm{d} \mathscr{A}_{\bar{\theta}}\right] \exp \left(-\frac{\mathrm{i} k}{2 \pi} \int \mathrm{d} z \wedge \mathrm{d} \bar{z} \int \mathrm{d}^{2} \theta\left(\mathscr{A}_{\theta}, \mathscr{A}_{\bar{\theta}}\right)\right) \Psi^{(1)}\left[\mathscr{A}_{\bar{\theta}}\right]^{*} \Psi^{(2)}\left[\mathscr{A}_{\bar{\theta}}\right],
$$

where the functional integral is over all superfields $\mathscr{A}_{\theta}, \mathscr{A}_{\bar{\theta}}$ on the surface $\Sigma$. Using (18) and (4), this is seen to be gauge invariant. The reduction of the inner product from the full phase space to the reduced phase space in the manner of Elitzur et al. [2] remains to be carried out; due to the supersymmetry, it is expected that there will be no renormalization of $k$ from jacobian factors.

As the hamiltonian vanishes weakly, the partition function on $\Sigma \times S^{1}$ is just a (super) trace over the Hilbert space of states. Using (6) and (7) and the identification of $\Psi\left[\mathscr{A}_{\theta}\right]$ with $\exp \left(-\Gamma_{+}\left[\mathscr{A}_{\theta}\right]\right)$, this is equivalent to the partition function for the gauged SWZW theory. Also, in the case $\partial \Sigma$ vanishes, the action (11) differs from the symmetric version presented at the end of section 3 by $-(k / 4 \pi) \int \mathrm{d} t \int \mathrm{d} z \wedge \mathrm{d} z \int \mathrm{d}^{2} \theta \partial_{t}\left(\mathscr{A}_{\theta}, \mathscr{A}_{\bar{\theta}}\right)$, so the trace 
can be considered as the result of a functional integral on $\Sigma \times S^{1}$ for the symmetric version of the action. This is related to the interpretation of wavefunctions as resulting from functional integrals over bounded manifolds.

(iii) As has been stressed, the "time" coordinate in the action (11) really parameterizes a one-parameter family of superconnections $\mathscr{A}_{\theta}$ and $\mathscr{A}_{\bar{\theta}}$ on $\Sigma$ (as is done when relating $2 \mathrm{D}$ nonabelian anomalies to ChernSimons terms). Accordingly, the fermions in the theory are 2D spinors rather than $3 \mathrm{D}$ spinors, so the theory does not possess 3D Lorentz invariance. This makes the extension of the definition of the action (11) to manifolds other than $\Sigma \times \mathbb{R}$ or $\Sigma \times S^{1}$ problematic, as would be necessary to calculate partition functions on arbitrary three-manifolds using the functional integral.

Despite this, there is still the hope that the partition function for the action (11) on arbitrary three-manifolds can be computed using canonical techniques. As has been stressed by Witten in his original work [1], an arbitrary three-manifold can be generated from $S^{3}$ by a sequence of surgery. Surgery means cutting a tube (whose surface has the topology of a torus) from a three-manifold and replacing it after making a modular transformation on the surface of the tube. This should allow the computation of the partition function of the theory for any three-manifold from a knowledge of the Hilbert space associated with the torus and the action of the modular group on this space [1]. The latter can be obtained from the known (at least in the split case) action of the modular group on the SWZW superconformal blocks.

(iv) All the discussion in section 4 and section 5 was for the case of a split super Riemann surface, where there is no nontrivial gravitino background and the super Riemann surface reduces to an ordinary Riemann surface and a choice of spin structure. However, for certain choices of spin structure, it is possible to include nontrivial gravitino backgrounds (corresponding to super Teichmüller parameters). This would considerably complicate the structure discussed in section 4 , leading to a mixing of the vector spaces $\wedge^{i} \mathrm{~V}(u)$ for different $i$. In terms of the SWZW superconformal blocks, this reflected in the failure of super Kac-Moody characters to factorize into an ordinary Kac-Moody and a free-fermion partition function [25].

\section{References}

[1] E. Witten, Commun. Math. Phys. 121 (1989) 351.

[2] M. Bos and V.P. Nair, Phys. Lett. B 223 (1989) 61; S.E. Elitzur, G. Moore, A. Schwimmer and N. Seiberg, Nucl. Phys. B 326 (1989) 108; G. Moore and N. Seiberg, Phys. Lett. B 220 (1989) 422.

[3] E. Verlinde, Nucl. Phys. B 300 (1988) 360.

[4] N. Sakai and T. Tanii, Prog. Theor. Phys. 83 (1990) 968.

[5] S. Carlip and I. Kogan, Mod. Phys. Lett. A 6 (1991) 171.

[6] L. Alvarez-Gaumé and P. Ginsparg, Nucl. Phys. B 243 (1984) 449; M. Atiyah and I. Singer, Proc. Natl. Acad. Sci. USA 81 (1984) 2597.

[7] E. Guadagnini, K. Konishi and M. Mintchev, Phys. Lett. B 157 (1985) 37; G. Girardi, R. Grimm and R. Stora, Phys. Lett. B 156 (1985) 203;

I.N. McArthur and H. Osborn, Nucl. Phys. B 268 (1986) 573.

[8] D. Friedan, in: Unified string theories, eds. M. Green and D. Gross (World Scientific, Singapore, 1986); G. Moore and P. Nelson, Nucl. Phys. B 274 (1986) 509.

[9] P. di Vecchia, V.G. Knizhnik, J.L. Petersen and P. Rossi, Nucl. Phys. B 253 (1985) 701; T.L. Curtright and C.K. Zachos, Phys. Rev. Lett. 53 (1984) 1799.

[10] E. Witten, Commun. Math. Phys. 92 (1984) 455.

[11] A.M. Polyakov and P.B Wiegmann, Phys. Lett. B 131 (1983) 121.

[12] M. Rakowski and G. Thompson, Phys. Lett. B 220 (1989) 557.

[13] P. di Vecchia, B. Durhuus and J.L. Petersen, Phys. Lett. B 144 (1984) 245.

[14] K. Gawedzki and A. Kupiainen, Nucl. Phys. B 320 (1989) 625.

[15] K. Gawedzki, Nucl. Phys. B 328 (1989) 733.

[16] H. Murayama, Z. Phys. C 48 (1990) 79.

[17] E. Verlinde and H. Verlinde, Conformal field theory and geometric quantization, Princeton University preprint PUPT-89/1149.

[18] J. Wess and B. Zumino, Phys. Lett. B 37 (1971) 95. 
[19] M. Henneaux, Classical foundations of BRST symmetry (Bibliopolis, Naples, 1988).

[20] D. Friedan and S. Shenker, Nucl. Phys. B 281 (1987) 509.

[21] M.F. Atiyah and R. Bott, Philos. Trans. R. Soc. London A 308 (1982) 523.

[22] L. Alvarez-Gaumé, G. Moore and C. Vafa, Commun. Math. Phys. 106 (1986) 1.

[23] E. Witten, J. Diff. Geom. 17 (1982) 661.

[24] V.G. Kac and I.T. Todorov, Commun. Math. Phys. 102 (1985) 337.

[25] I.N. McArthur, Nucl. Phys. B 340 (1990) 148. 